\title{
Behavioural activation for depression in older people: systematic review and meta-analysis
}

Vasiliki Orgeta, Janina Brede and Gill Livingston

\section{Background}

Behavioural activation is an effective treatment for depression in the general adult population but it is unclear whether it is effective for older people.

\section{Aims \\ To systematically review randomised controlled trials (RCTs) of behavioural activation for depression in older people.}

\section{Method}

We searched Medline, EMBASE, PsycINFO, CINAHL and online trial registers for RCTS of behavioural activation for depression in older people.

\section{Results}

Eighteen trials were included in the meta-analyses Behavioural activation reduced mean depression scores for older people living in the community as a stand-alone treatment: standardised mean difference (SMD) $-0.72,95 \%$ $\mathrm{Cl}-1.04$ to -0.41 . It was also effective as part of a multicomponent intervention (SMD $-0.44,95 \% \mathrm{Cl}-0.56$ to $-0.32)$.

\section{Conclusions}

Behavioural activation significantly reduces depressive symptoms in older people in the community; however, given that most studies are small and with significant bias results should be interpreted with caution. Further high-quality trials of behavioural activation for this age group are needed.

\section{Declaration of interest}

V.O. and G.L. are principal investigators of an ongoing trial.

\section{Copyright and usage}

(c) The Royal College of Psychiatrists 2017.
Depression in older people is a prevalent and disabling condition, with major depressive disorder affecting approximately $5-15 \%$ of older people living in the community. ${ }^{1}$ Estimates of prevalence of mild symptoms of depression, often referred to as subsyndromal depression, are much higher, ranging from $5 \%$ to $37 \% .{ }^{2}$ Untreated symptoms - even when mild - can contribute to poor physical and psychiatric outcomes such as increasing the number and severity of episodes of medical illness and the risk of developing major depression. ${ }^{3}$ Depression diminishes older adults' quality of life, exacerbates functional deficits and increases risk of morbidity and mortality. ${ }^{4}$ It is the major contributor to poor outcomes in older people, and contributes heavily to healthcare costs. $^{5}$ Depression in late life is often underdetected and untreated, ${ }^{1}$ and may be resistant to antidepressant therapy. ${ }^{6}$ Many studies of psychological treatments have few or no older participants. It is estimated that preventing and treating depressive symptoms in older people may result in significantly reduced healthcare use and in cost savings. ${ }^{7}$ With an increasingly ageing population it is important to find out what strategies are effective for managing depression in older people and addressing the needs of those with cognitive impairment. Behavioural explanations of low mood and depressive affect are rooted in learning theory models of depression, which argue that increasing positively reinforcing behaviours leads to more positive consequences for individuals, thereby improving mood. ${ }^{8}$ The lack of events associated with positive outcomes in the person's environment is a key contributor to the emergence or maintenance of depressive symptoms. ${ }^{9}$ According to behavioural models of depression in late life, ${ }^{10}$ depressive symptoms may be intensified or maintained by the absence of positive feelings resulting from participation in enjoyable and meaningful activity. Interventions that target activities may therefore theoretically be particularly helpful in preventing or treating depression in older people. Behavioural activation is an effective treatment for depression for adults, with recent studies indicating that it is as effective as cognitivebehavioural therapy (CBT) and potentially less costly. ${ }^{11}$ Despite evidence of clinical effectiveness in younger adults, evidence of effectiveness in older people is less clear. Although there are several studies evaluating behavioural activation in older people, there is no systematic review bringing the evidence together. Our aim was to evaluate worldwide evidence on the clinical effectiveness of behavioural activation for depression in older people and report on its quality.

\section{Method}

We searched all related behavioural activation and behaviour therapy terms in Medline, EMBASE, CINAHL, PsycINFO and the Cochrane library for ongoing trials, national and international trial registers, and specialised databases of psychological treatments of depression (www.evidencebasedpsychotherapies.org) to December 2016 (see online supplement DS1 for details of search terms). We scanned reference lists of all included studies and 136 reviews relevant to the area identified by the search (psychological therapies in older people with or without cognitive impairment or dementia).

Inclusion criteria were as follows. Studies had to be randomised controlled trials (RCTs) comparing behavioural activation with treatment as usual (or any other treatment); samples comprised older people (at least 55 years old) in any setting (community, in-patient or long-term care) and included participants with or without cognitive impairment or dementia. Outcomes were either depressive symptom score or depression remission. Secondary outcomes were function, quality of life and anxiety symptoms.

We defined behavioural activation as a brief, structured psychotherapeutic approach that aimed to increase engagement in adaptive activities (often associated with pleasure and mastery) through structured activity scheduling and monitoring of mood (association of mood and activities), and/or other behavioural strategies such as relaxation and stress reduction techniques, social skills enhancement or hierarchical construction of goals. ${ }^{12}$ Some studies also included additional interventions. Activity interventions that did not satisfy these criteria or had a large exercise 
component were defined as behavioural activation-related. We divided interventions according to where they took place (community, in-patient $/ 24 \mathrm{~h}$ care settings, care homes) and by short-term (4-12 weeks) or long-term (8-12 months) effectiveness. We also tested whether publication bias was present.

Two reviewers (V.O. and J.B.) worked independently to identify RCTs that met the inclusion criteria, and extracted data independently. Disagreements were discussed with the third author (G.L.). We contacted authors of primary trials if there were missing data. We employed the Cochrane Handbook for Systematic Reviews of Interventions approach for assessing risk of bias, addressing the domains of sequence generation, allocation concealment, masking, incomplete outcome data, selective reporting and other issues (www.handbook.cochrane.org).

\section{Statistical analysis}

We used a random effects model to represent overall estimate effects, and standardised mean differences (SMDs) when studies used different outcome scales. We quantified heterogeneity using the $I^{2}$ statistic. All calculations were conducted using Review Manager (RevMan 5.2) for Windows. We used the Grading of Recommendations Assessment, Development and Evaluation (GRADE) approach to summarise overall quality of evidence (www.gradeworkinggroup.org), and performed a sensitivity meta-analysis on high-quality studies only.

\section{Results}

We identified 7248 references through database searching, with 33 additional references identified through other sources (Fig. 1). After removal of duplicates and clearly irrelevant articles, we retrieved 391 full-text records. Of these, 327 were excluded at this stage as not relevant, leaving 64 full-text references to be fully assessed for eligibility. Of these, 36 studies were excluded as not meeting inclusion criteria: they evaluated behavioural activation in family carers (8 studies); were not RCTs (14 studies); the intervention did not fulfil our criteria of behavioural activation (12 studies); there was no control comparison group (1 study); and data were not available ( 1 study). A total of 28 studies were eligible for inclusion, of which 7 were ongoing. ${ }^{13-19}$ The main characteristics of the studies, including descriptions of elements of interventions, are summarised in online Tables DS1 (included studies) and DS2 (excluded studies).

\section{Description of studies}

We were able to pool data from 18 studies. There were nine studies in older people living in the community, three RCTs in in-patient settings and six studies in older people living in care homes, some of whom had cognitive impairment. We pooled data from five RCTs on behavioural activation only (community settings), ${ }^{20-24}$ and four trials on multicomponent behavioural activation (community settings), ${ }^{25-28}$ for depressive symptoms. All of the multicomponent behavioural activation studies provided data on remission of symptoms. We pooled data from all three RCTs evaluating behavioural activation in in-patient settings, ${ }^{29-31}$ and all six studies of behavioural activation in older people living in care homes. ${ }^{32-37}$ Although there were three studies of people with dementia that aimed to increase activity, none of these met the inclusion criteria. These studies were in community-dwelling people with dementia, and evaluated interventions with a strong physical activity component, and activity scheduling was not structured; ${ }^{38-40}$ we classified them as behavioural activation-related. We judged these studies as too different to be

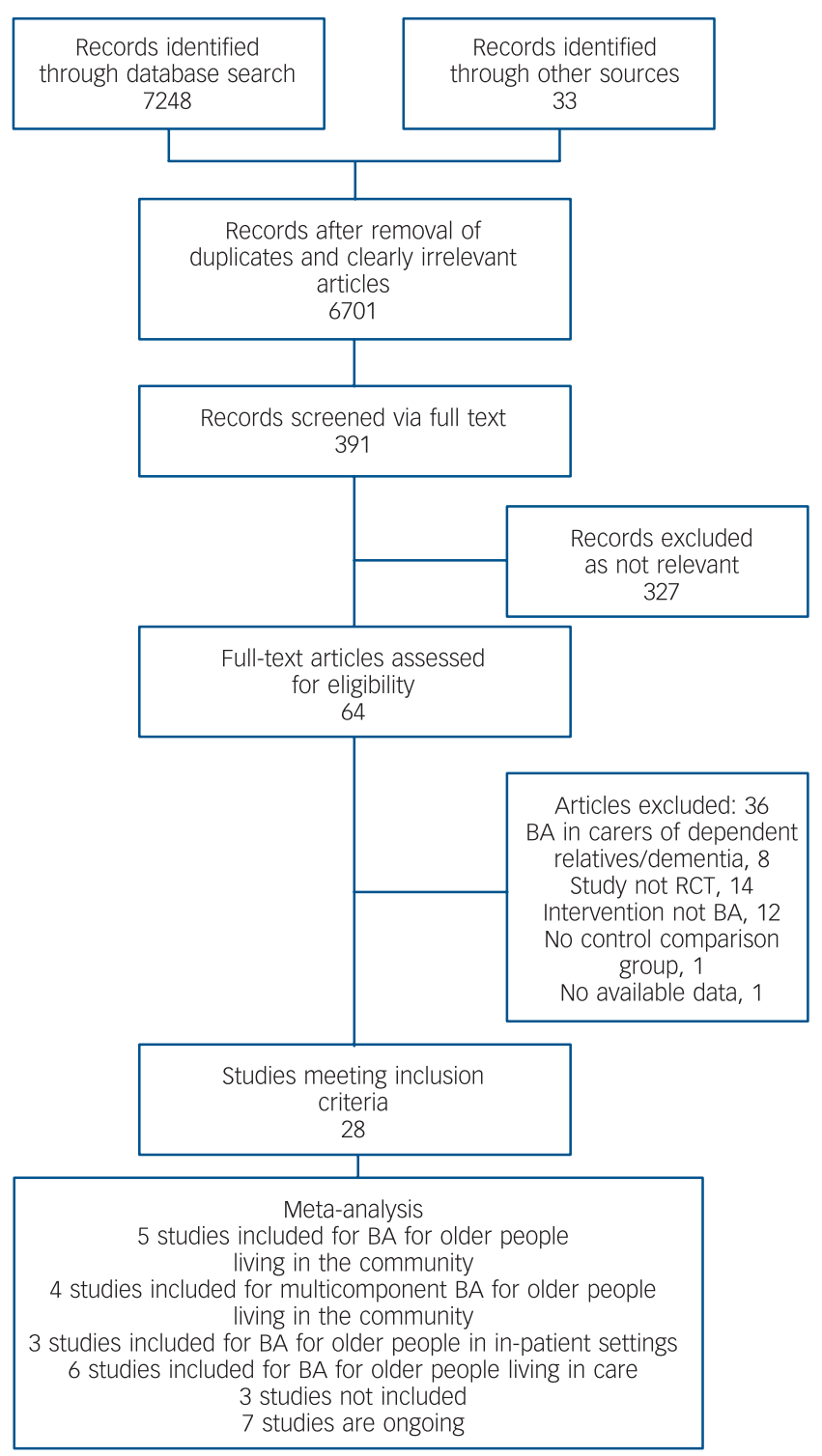

Fig. 1 Study flow diagram. BA, behavioural activation; $\mathrm{RCT}$, randomised controlled trial.

pooled in a meta-analysis. We used the chi-squared test to detect and report on heterogeneity.

\section{Primary outcomes}

\section{Depression severity}

Meta-analysis of the effects of behavioural activation on depressive symptoms showed that results significantly favoured the therapy (5 studies; $n=175, \mathrm{SMD}=-0.72,95 \%$ CI -1.04 to -0.41 , efficacy at 4-12 weeks) in reducing depressive symptoms for older people living in the community (with a diagnosis of depression or subthreshold depressive symptoms), with no heterogeneity between the studies $\left(I^{2}=0 \%\right.$ ) (Fig. 2). Multicomponent behavioural activation reduced depressive symptoms in comparison with treatment as usual in the short term (4 studies; $n=2523$, $\mathrm{SMD}=-0.44,95 \% \mathrm{CI}-0.56$ to -0.32 , efficacy at 3-6 months; Fig. 3) but not long-term (4 studies; $n=2300, \mathrm{SMD}=-0.30,95 \%$ CI -0.59 to 0.00 , efficacy at $8-12$ months; Fig. 4). There was moderate $\left(I^{2}=39 \%\right)$ to high heterogeneity $\left(I^{2}=88 \%\right)$ in both of these analyses. 
Older people with depression diagnosis

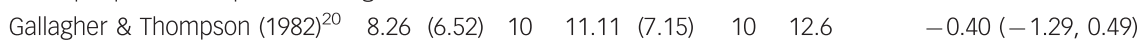

Thomspon \& Gallagher $(1984)^{21} 11.63(7.67) \quad 18 \quad 16.67(4.9) \quad 9 \quad 14.6 \quad-0.71(-1.53,0.12)$

Subtotal $(95 \% \mathrm{Cl}) \quad 28 \quad 19 \quad 27.2 \quad-0.56(-1.17,0.04)$

Heterogeneity: $\tau^{2}=0.00 ; \chi^{2}=0.25$, d.f. $=1(P=0.62) ; I^{2}=0 \%$

Test for overall effect: $Z=1.83(P=0.07)$

Older people with depressive symptoms

Cernin \& Lichtenberg (2009) $)^{22} \quad 8.9 \quad(5) \quad 8 \quad 12.7 \quad 6.5$

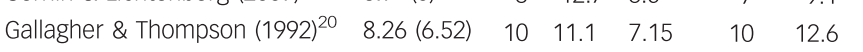

$\begin{array}{lllllll}\text { Moss et al }(2012)^{23} & 5.46(4.96) & 10 & 10.85 & 4.96 & 13 & 12.6\end{array}$

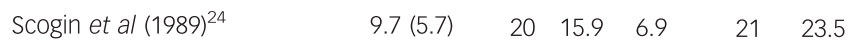

$\begin{array}{lllllll}\text { Thompson \& Gallagher }(1984)^{21} & 11.63(7.67) & 20 & 16.67 & 4.9 & 9 & 15.1\end{array}$

$\begin{array}{llll}\text { Subtotal }(95 \% \mathrm{Cl}) & 68 & 60 & 72.8\end{array}$

Heterogeneity: $\tau^{2}=0.00 ; \chi^{2}=1.47$, d.f. $=4(P=0.83) ; I^{2}=0 \%$

Test for overall effect: $Z=4.15(P<0.0001)$

Total $(95 \% \mathrm{Cl})$

Heterogeneity: $\tau^{2}=0.00 ; \chi^{2}=2.08$, d.f. $=6(P=0.91) ; I^{2}=0 \%$

Test for overall effect: $Z=4.50(P<<0.001)$

Test for subgroup differences: $\chi^{2}=0.36$, d.f. $=1(P=0.55) ; I^{2}=0 \%$

$79100.0 \quad-0.72(-1.04,-0.41)$

$-0.62(-1.67,0.42)$

$-0.40(-1.29,0.49)$

$-1.05(-1.94,-0.16)$

$-0.96(-1.61,-0.31)$

$-0.70(-1.51,0.11)$

$-0.78(-1.15,-0.41)$

, $95 \% \mathrm{Cl}$

Fig. 2 Forest plot of behavioural activation (all forms) $v$. treatment as usual for older people living in the community with a diagnosis of depression or depressive symptoms. Outcome: depressive symptoms (4-12 weeks). SMD, standardised mean difference.

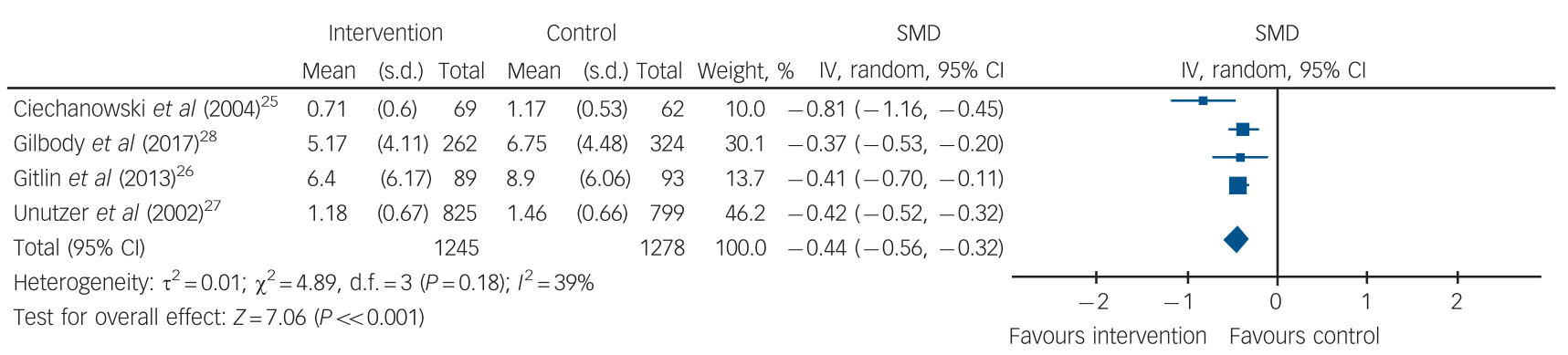

Fig. 3 Forest plot of multicomponent behavioural activation $v$. treatment as usual for older people living in the community with a diagnosis of depression or depressive symptoms. Outcome: depressive symptoms (3-6 months). SMD, standardised mean difference.

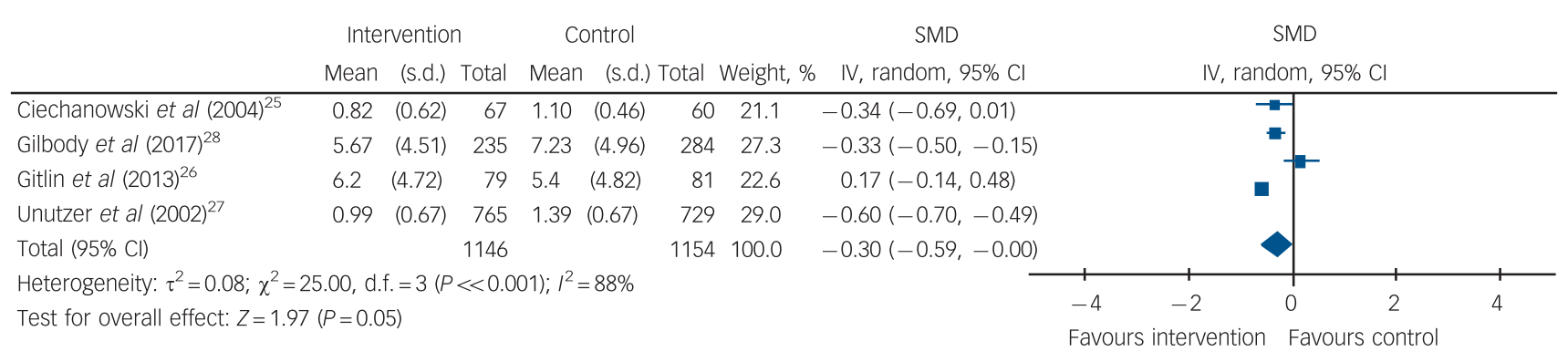

Fig. 4 Forest plot of multicomponent behavioural activation v. treatment as usual for older people living in the community with a diagnosis of depression or depressive symptoms. Outcome: depressive symptoms (8-12 months). SMD, standardised mean difference.

\section{Remission of symptoms}

Multicomponent behavioural activation improved remission of depressive symptoms, both in the short term (4 studies; $n=2733$, odds ratio $(\mathrm{OR})=2.66,95 \%$ CI 1.46 to 4.87 , efficacy at 3-6 months, $I^{2}=81 \%$; online Fig. DS1) and long term (3 studies; $n=2458, \mathrm{OR}=3.06,95 \%$ CI 1.94 to 4.82 , efficacy at $8-12$ months, $I^{2}=63 \%$; online Fig. DS2).

\section{Care setting}

We found no significant difference between the intervention and treatment as usual groups for older people in psychiatric in-patient settings ( 3 studies; $n=74, \mathrm{SMD}=-0.11,95 \% \mathrm{CI}-0.57$ to 0.35 , efficacy at 2-8 weeks, $I^{2}=0 \%$ ) or long-term care settings ( 6 studies; 302 participants with and without dementia, $\mathrm{SMD}=-0.43,95 \% \mathrm{CI}$ -0.87 to 0.01 , efficacy at $6-12$ weeks, $I^{2}=65 \%$ ). 


\section{Secondary outcomes}

Multicomponent behavioural activation improved quality of life compared with treatment as usual (2 studies; $n=1806$, $\mathrm{SMD}=0.33,95 \%$ CI 0.12 to 0.54 , efficacy at 3-6 months, $\left.I^{2}=54 \%\right)$ and decreased functional disability for older people living in the community ( 2 studies; $n=1806, \mathrm{SMD}=-0.24$, $95 \%$ CI -0.33 to -0.15 , efficacy at $3-6$ months, $I^{2}=0 \%$ ). There was a significant effect of multicomponent behavioural activation, in comparison with treatment as usual, on reduction of anxiety symptoms in the short term ( 2 studies; $n=761, \mathrm{SMD}=-0.30$ $95 \%$ CI -0.44 to -0.15 , efficacy at 4 months, $I^{2}=0 \%$ ), which was not maintained at long-term follow-up ( 2 studies; $n=739$, $\mathrm{SMD}=-0.16,95 \% \mathrm{CI}-0.38$ to 0.06 , efficacy at $8-12$ months, $\left.I^{2}=43 \%\right)$. None of the studies reported or described adverse events.

\section{Quality of studies}

Bias was detected predominantly in the domains of sequence generation and allocation concealment, with one study being at high risk in the domain of masking of outcome assessment (for detailed ratings see online Fig. DS3). We assessed publication bias with a funnel plot (online Fig. DS4) which appeared to be approximately symmetrical, indicating no association between standardised effect size and standard errors of effects, confirmed by Egger's test $(t=-1.326, P=0.203)$. Using the GRADE approach, we judged the overall quality of evidence of effectiveness of behavioural activation for depressive symptoms as moderate. We also considered the potential bias and overestimation of effects by running analyses only on high-quality studies as judged by predetermined criteria used in previous meta-analyses of psychotherapy trials. ${ }^{41}$ Only studies of multicomponent behavioural activation (4 studies) and behavioural activation in care homes (3 studies) qualified to be included in the analyses: $\mathrm{SMD}=-0.40$, $95 \%$ CI -0.48 to $-0.32, I^{2}=48 \%$ (7 studies, $n=2676$ ).

\section{Discussion}

We aimed to establish the most up-to-date and accurate estimate of the effectiveness of behavioural activation for older people. We also evaluated the quality of the evidence and presence of publication bias. We found that overall there was evidence of effects for this intervention in the range usually regarded as clinically significant, ${ }^{42}$ with SMD values of $0.40-1.05$ for behavioural activation alone and $0.37-0.81$ for multicomponent behavioural activation, for older people living in the community. When only high-quality studies were considered the effect size was still 0.4 . Five RCTs with a total of 175 participants ( 96 receiving behavioural activation, 79 treatment as usual) showed that this intervention reduced depressive symptoms in community-dwelling older people. These results compare favourably with studies of CBT for depression in older people, and are in line with recent data on the comparable effectiveness of behavioural activation in all adults. ${ }^{11}$ However, given the paucity of trials and methodological limitations, there is still some uncertainty about this result. Four studies showed that multicomponent behavioural activation approaches benefit older people in the community with symptom remission in the short term and also approximately a year later. In terms of symptom reduction, multicomponent approaches were effective only in the short term, with differences no longer being significant in the longer term, indicating that effects are less stable. Further studies are required to confirm these results. Our analyses did not find that adding other care components such as case management or collaborative care adds to the effectiveness of behavioural activation approaches, similar to evidence in adult depression. ${ }^{43}$ Only multicomponent approaches evaluated remission of symptoms. Although only a few studies were pooled for secondary outcomes, multicomponent behavioural activation improved functional outcomes, quality of life and anxiety in the short term. It will be important for future trials to assess long-term effectiveness of behavioural activation for older people as a stand-alone intervention.

Data on the effectiveness of behavioural activation in in-patient and long-term care settings did not show that it decreased depressive symptoms, but the majority of studies were small and of lower quality. We found three trials evaluating behavioural activationrelated interventions for people with dementia. These studies, however, did not employ theoretically driven and structured approaches of behavioural activation, or included strong components of physical activity, thereby limiting the conclusions of effectiveness of behavioural activation per se.

We found that the studies evaluating behavioural activation for older people differed in terms of inclusion criteria. Although using broad inclusion criteria can be an advantage in producing results that are more generalisable, samples varied between people with or without a depression diagnosis and by intervention delivery (by mental health workers $v$. self-help). Self-help behavioural activation may be an impractical intervention for the most severely depressed people, who are particularly lacking in energy and motivation and may be restricted in going out from care settings because of concerns about self-harm. Our multicomponent behavioural activation, in-patient and long-term care meta-analyses included studies with varied participants in terms of medical comorbidity and cognitive impairment. It has been argued that the complex interaction of medical morbidity, cognitive impairment and psychological factors make depression in late life difficult to treat. Therefore our findings are important in that they suggest that behavioural activation may be able to address the complexity of preventing and treating depressive symptoms in older people.

\section{Quality of the evidence}

Risk of bias was unclear for multiple domains in some of the studies, with published information sometimes insufficient to determine risk of bias. Only the area of selective reporting was judged as low risk in all studies. Most of the studies had uncertainties on the areas of random sequence generation and allocation concealment. Applying the GRADE system we classified the quality of the evidence as moderate. Although publication bias did not have a significant effect on the results, we found that the effects of behavioural activation may be overestimated when including small studies. However, our analyses were based on a limited number of trials overall, and we were unable to conduct other sensitivity analyses because of lack of power of potential comparisons.

\section{Limitations}

This review has several limitations. The studies had relatively low age criteria, with many including adults aged 55 years and over, whereas others had a minimum age criterion of 65 years. Many participants would therefore be less likely to have the typical circumstances of older people such as multiple physical comorbidities and no longer having employment. Our test of publication bias does not provide direct evidence of absence of bias, so it is likely that the studies overestimate the true effect size of this approach. Some of the studies investigated bibliotherapy as a treatment for late-life depressive symptoms, which may limit its 
use in some settings. Although all studies used central components of behavioural activation such as structured pleasant activity scheduling and mood monitoring, some reported on the use of additional behavioural strategies, which limits our understanding of which specific components are important. ${ }^{12}$ High heterogeneity may have contributed to our lack of group differences in some of the outcomes and the comparison with waiting-list control conditions in some of the studies may have overestimated the effects. We also could not find information on coexisting anxiety disorders. Although there was a wide range of depression severity within studies, this range may enable findings to be generalisable to a large proportion of older people who are likely to seek treatment.

\section{Implications for practice and research}

Our findings are in line with evidence that behavioural activation may be associated with medium to large effects, being observed in relatively few sessions delivered by junior mental health workers. ${ }^{11}$ Most of the studies included an average of 8-10 sessions across all settings, implemented primarily by non-mental health specialists. We also found that behavioural activation improved other outcomes such as quality of life and functional disability, and decreased anxiety symptoms in the short term. Currently, management of late-life depression with or without cognitive impairment is limited to the prescription of antidepressants, where there may be poor adherence or inconclusive evidence of effectiveness. ${ }^{6,44}$ Our data show that high-quality mental healthcare for treating or preventing clinically significant symptoms of depression in older people may benefit from individualised treatments that emphasise pleasant activities. There is a need, however, for well-designed multicentre RCTs that adhere to high standards of methodology and reporting. These trials should focus on theoretically driven behavioural activation interventions. The lack of follow-up data poses limitations in terms of informing evidence-based policy about how best to deliver behavioural activation for older people, and there are no data on cost-effectiveness. Future studies should examine longterm effects. There remains a need for carefully designed studies of interventions for older people with cognitive impairment and dementia, and considering evaluation of behavioural activation separately for older people with and without cognitive impairment, as findings may differ.

\section{Future research}

On the basis of this meta-analysis we conclude that although behavioural activation may be a potentially effective treatment for mild or subthreshold symptoms and major depression in older people, evidence remains limited. Our review suggests that additional components such as care collaboration or case management may not add to effectiveness. Given the small number of RCTs and presence of bias in studies, effects associated with behavioural activation should be interpreted with caution. Future large-scale studies are necessary to establish the effectiveness of these approaches in older people.

Vasiliki Orgeta, PhD, Janina Brede, MSC, Gill Livingston, FRCPsych, MD, Division of Psychiatry, University College London, UK

Correspondence: Dr Vasiliki Orgeta, Division of Psychiatry, University College London, 6th Floor, Maple House, 149 Tottenham Court Road, London W1T 7NF, UK. Email: v.orgeta@ucl.ac.uk

First received 17 Feb 2017, final revision 27 Jun 2017, accepted 3 Jul 2017

\section{Funding}

We thank the Alzheimer's Society for their funding of the work undertaken.

\section{Acknowledgements}

We thank all the authors who provided data and further information for this review.

\section{References}

1 Rodda J, Walker Z, Carter J. Depression in older adults. BMJ 2011; 343: d5219.

2 Meeks TW, Vahia IV, Lavretsky H, Kulkarni G, Jeste DV. A tune in 'a minor' can 'b major': a review of epidemiology, illness course, and public health implications of subthreshold depression in older adults. J Affect Disord 2011; 129: $126-42$.

3 Koenig HG. Differences in psychosocial and health correlates of major and minor depression in medically ill older adults. J Am Geriatr SoC 1997; 45: 1487-95.

4 Schoevers RA, Geerlings MI, Beekman AT, Penninx BW, Deeg DJ, Jonker C, et al. Association of depression and gender with mortality in old age. Results from the Amsterdam Study of the Elderly (AMSTEL). Br J Psychiatry 2000; 177: 336-42.

5 Vasiliadis HM, Dionne PA, Preville M, Gentil L, Berbiche D, Latimer E. The excess healthcare costs associated with depression and anxiety in elderly living in the community. Am J Geriatr Psychiatry 2013; 21: 536-48.

6 Knochel C, Alves G, Friedrichs B, Schneider B, Schmidt-Rechau A, Wenzler S, et al. Treatment-resistant late-life depression: challenges and perspectives. Curr Neuropharmacol 2015; 13: 577-91.

7 Smit F, Ederveen A, Cuijpers P, Deeg D, Beekman A. Opportunities for costeffective prevention of late-life depression: an epidemiological approach. Arch Gen Psychiatry 2006; 63: 290-6.

8 Lewinsohn PM, Hoberman HM, Teri L, Hautzinger M. An integrative theory of depression. In Theoretical Issues in Behavior Therapy (eds S Reiss, RR Bootzin): 331-59. Academic Press, 1985.

9 Lewinsohn PM, Munoz RF, Youngren MA, Zeis AM. Control Your Depression. Prentice Hall, 1986.

10 Fiske A, Wetherell JL, Gatz M. Depression in older adults. Annu Rev Clin Psychol 2009; 5: 363-89.

11 Richards DA, Ekers D, McMillan D, Taylor RS, Byford S, Warren FC, et al. Cost and Outcome of Behavioural Activation versus Cognitive Behavioural Therapy for Depression (COBRA): a randomised, controlled, non-inferiority trial. Lancet 2016; 388: 871-80.

12 Dimidjian S, Barrera M, Martell C, Munoz RF, Lewinsohn PM. The origins and current status of behavioral activation treatments for depression. Annu Rev Clin Psychol 2011; 7: 1-38.

13 Farrand P. Behavioural activation self-help for well-being in people with dementia (ISRCTN42017211). ISRCTN Registry, 2015 (www.isrctn.com).

14 Raue P. Peer to peer delivery of behavioral activation (NCT02292849). 2014, ClinicalTrials.gov.

15 Clignet F, van Meijel B, van Straten A, Cuijpers P. The systematic activation method as a nursing intervention in depressed elderly: a protocol for a multicentre cluster randomized trial. BMC Psychiatry 2012; 12: 144.

16 Orgeta V. IDEA: Intervention to prevent depressive symptoms and promote well-being in early stage dementia (ISRCTN75503960). ISRCTN Registry, 2015 (www.isrctn.com).

17 Overend $K$, Lewis $H$, Bailey D, Bosanquet $K$, Chew-Graham C, Ekers D, et al. CASPER plus (CollAborative care in Screen-Positive EldeRs with major depressive disorder): study protocol for a randomised controlled trial. Trials 2014; 15: 451.

18 Mitchell N, Hewitt C, Adamson J, Parrott S, Torgerson D, Ekers D, et al. A randomised evaluation of CollAborative care and active surveillance for Screen-Positive EldeRs with sub-threshold depression (CASPER): study protocol for a randomized controlled trial. Trials 2011; 12: 225.

19 Dong $X$, Chen R, Wong E, Simon MA. Suicide prevention in Chinese older adults (NCT02096432). 2014, ClinicalTrials.gov.

20 Gallagher DE, Thompson LW. Treatment of major depressive disorder in older adult outpatients with brief psychotherapies. Psychotherapy: Theory, Research and Practice 1982; 19: 482-90.

21 Thompson LW, Gallagher D. Efficacy of psychotherapy in the treatment of late-life depression. Adv Behav Res Ther 1984; 6: 127-39. 
22 Cernin PA, Lichtenberg PA. Behavioral treatment for depressed mood: a pleasant events intervention for seniors residing in assisted living. Clin Gerontol 2009; 32: 324-31.

23 Moss K, Scogin F, Di Napoli E, Presnell A. A self-help behavioral activation treatment for geriatric depressive symptoms. Aging Ment Health 2012; 16 : 625-35

24 Scogin F, Jamison C, Gochneaur K. Comparative efficacy of cognitive and behavioral bibliotherapy for mildly and moderately depressed older adults. J Consult Clin Psychol 1989; 57: 403-7.

25 Ciechanowski $\mathrm{P}$, Wagner E, Schmaling K, Schwartz S, Williams B, Diehr P, et al. Community-integrated home-based depression treatment in older adults: a randomized controlled trial. JAMA 2004; 291: 1569-77.

26 Gitlin LN, Harris LF, McCoy MC, Chernett NL, Pizzi LT, Jutkowitz E, et al. A home-based intervention to reduce depressive symptoms and improve quality of life in older African Americans: a randomized trial. Ann Intern Med 2013; 159: 243-52.

27 Unutzer J, Katon W, Callahan CM, Williams JW, Hunkeler E, Harpole L, et al. Improving mood-promoting access to collaborative treatment. collaborative care management of late-life depression in the primary care setting: a randomized controlled trial. JAMA 2002; 288: 2836-45.

28 Gilbody S, Lewis H, Adamson J, Atherton K, Bailey D. Effect of collaborative care vs usual care on depressive symptoms in older adults with subthreshold depression: the CASPER randomized clinical trial. JAMA 2017; 317: 728-37.

29 Norton MJ. Evaluation of a Brief Behavioral Activation Therapy for Depression (BATD) group protocol in an inpatient geriatric psychiatry facility. Dissertation Abstracts International: Section B: The Sciences and Engineering 2010; 71: 7099

30 Snarski M, Scogin F, DiNapoli E, Presnell A, McAlpine J, Marcinak J. The effects of behavioral activation therapy with inpatient geriatric psychiatry patients. Behav Ther 2011; 42: 100-8.

31 Sood JR, Cisek E, Zimmerman J, Zaleski EH, Fillmore HH. Treatment of depressive symptoms during short-term rehabilitation: an attempted replication of the DOUR project. Rehabil Psychol 2003; 48: 44-9.

32 Dozeman E, van Schaik DJ, van Marwijk HW, Stek ML, Beekman AT, van der Horst HE. Feasibility and effectiveness of activity-scheduling as a guided self-help intervention for the prevention of depression and anxiety in residents in homes for the elderly: a pragmatic randomized controlled trial. Int Psychogeriatr 2011; 23: 969-78.
33 Hyer L, Yeager CA, Hilton N, Sacks A. Group, individual, and staff therapy: an efficient and effective cognitive behavioral therapy in long-term care. Am J Alzheimers Dis Other Demen 2008; 23: 528-39.

34 Lichtenberg PA, Kemp-Havican J, Macneill SE, Schafer Johnson A. Pilot study of behavioral treatment in dementia care units. Gerontologist 2005; 45 : 406-10.

35 Meeks S, Looney SW, Van Haitsma K, Teri L. BE-ACTIV: a staff-assisted behavioral intervention for depression in nursing homes. Gerontologist 2008; 48: $105-14$

36 Meeks S, Van Haitsma K, Schoenbachler B, Looney SW. BE-ACTIV for depression in nursing homes: primary outcomes of a randomized clinical trial. J Gerontol B Psychol Sci Soc Sci 2015; 70: 13-23.

37 Verkaik R, Francke AL, van Meijel B, Spreeuwenberg PM, Ribbe MW, Bensing JM. The effects of a nursing guideline on depression in psychogeriatric nursing home residents with dementia. Int J Geriatr Psychiatry 2011; 26: 723-32.

38 Teri L, Logsdon RG, Uomoto J, McCurry SM. Behavioral treatment of depression in dementia patients: a controlled clinical trial. J Gerontol B Psychol Sci Soc Sci 1997; 52: P159-66.

39 Teri L, Gibbons LE, McCurry SM, Logsdon RG, Buchner DM, Barlow WE, et al Exercise plus behavioral management in patients with Alzheimer disease: a randomized controlled trial. JAMA 2003; 290: 2015-22.

40 Gitlin LN, Winter L, Dennis MP, Hodgson N, Hauck WW. A biobehavioral home-based intervention and the well-being of patients with dementia and their caregivers: the COPE randomized trial. JAMA 2010; 304: 983-91.

41 Cuijpers $P$, van Straten A, Bohlmeijer E, Hollon SD, Andersson G. The effects of psychotherapy for adult depression are overestimated: a meta-analysis of study quality and effect size. Psychol Med 2010; 40: 211-23.

42 Kirsch I, Deacon BJ, Huedo-Medina TB, Scoboria A, Moore TJ, Johnson BT. Initial severity and antidepressant benefits: a meta-analysis of data submitted to the Food and Drug Administration. PLOS Med 2008; 5: e45.

43 National Collaborating Centre for Mental Health. Depression: The NICE Guideline on the Treatment and Management of Depression in Adults. Updated Edition. British Psychological Society/Royal College of Psychiatrists 2010

44 Banerjee S, Hellier J, Dewey M, Romeo R, Ballard C, Baldwin R, et al. Sertraline or mirtazapine for depression in dementia (HTA-SADD): a randomised, multicentre, double-blind, placebo-controlled trial. Lancet 2011; 378: 403-11. 